\title{
POLÍTICAS DE BIENESTAR EN CONTEXTOS NEOLIBERALES: tensiones del modelo chileno' ${ }^{1}$ POLÍTICAS DE BEM-ESTAR EM CONTEXTOS NEOLIBERAIS: tenções do modelo chileno ${ }^{1}$
}

\begin{abstract}
María Pía Martin*
Jaime Alfaro * *

Las sociedades latinoamericanas contemporáneas se han visto tensionadas por los conflictos entre múltiples visiones sobre la articulación entre crecimiento económico y políticas sociales. Este artículo expone el análisis desde el enfoque de marcos interpretativos que, desde la perspectiva constructivista, permite profundizar en la dimensión simbólica y representacional de las políticas. A partir de reflexiones teóricas y análisis sobre las visiones en la protección social en discusiones parlamentarias en el caso de Chile, la finalidad es aportar a una perspectiva de análisis que permita indagar en los procesos de cambio de las políticas sociales, y mostrar cómo el eje en las ideas ofrece una mejor comprensión de la estabilidad y el cambio de las políticas sociales.

Palabras Clave: Protección Social. Estado de Bienestar. Neoliberalismo. Análisis de Marcos Interpretativos. Políticas Públicas.

As sociedades latino-americanas contemporâneas se viram tensionadas por conflitos entre múltiplas visões sobre a articulação entre crescimento econômico e políticas sociais. Este artigo apresenta a análise de quadros interpretativos que, a partir da perspectiva construtivista, permitem aprofundar a dimensão simbólica e representativa das políticas. A partir de reflexões teóricas e análises sobre as visões de proteção social em discussões parlamentares, no caso do Chile, a finalidade é trazer uma perspectiva de análise que permita indagar sobre os processos de mudança das políticas sociais e mostrar como o eixo, nas ideias, oferece uma compreensão melhor da estabilidade e da mudança das políticas sociais.
\end{abstract}

Palavras-chave: Proteção Social. Estado de bem-estar. Neoliberalismo. Análise de quadros interpretativos. Políticas Públicas.

\section{INTRODUCCIÓN}

En los últimos años, en América Latina, ha habido un fortalecimiento de las políticas sociales, que pasan a tener un rol central en las agendas de los gobiernos. Ello se realiza en un contexto de discusión sobre los modos de concebir al Estado, las concepciones sobre noción de problema y noción de soluciones de intervención, la profundidad, el impacto y la cobertura de los cambios, y, en lo fundamental, cuánto de cambio o de continuidad existe respecto del ideario de focalización neoliberal. Por otra parte, diversos procesos, como la estabilización de las democracias, los mejores niveles de desarrollo económico, la extensión de

\footnotetext{
* Universidad de Chile. Departamento de Ingeniería Industrial. República,701.Santiago - Chile.mpmartin@dii.uchile.cl ** Universidad del Desarrollo. Departamento de Psicologia. Av. La Plaza 680. Santiago - Chile. jalfaro@udd.cl

${ }^{1}$ Este trabajo se origina en el Proyecto FONDECYT de Investigación Regular N ${ }^{\circ} 1150938$, "La configuración del campo técnico del psicólogo en la política social: análisis de la formulación e implementación de programas sociales con componente psicosocial.
}

los Programas de Transferencia Condicionadas $(\mathrm{PTC})^{2}$ y las propias deficiencias del modelo asistencialista y neoliberal impulsado por los organismos internacionales a raíz del Consenso de Washington, ${ }^{3}$ marcan el contexto de la emergencia de una nueva agenda social en la región. Si, a inicios de los noventa, las principales discusiones en América Latina se establecían en torno a los procesos de recuperación de la democracia y los desafíos marcados por el cambio desde sistemas autoritarios a gobiernos democráticos, actualmente el cambio democrático no sólo pone en la agenda los temas

${ }^{2}$ Los Programas de Transferencia Condicionada (PTC) se han implementado masivamente en América Latina. Buscan superar la extrema pobreza mediante el desarrollo de las capacidades y activación de capital humano. Entregan transferencias monetarias directas en función de ciertas condicionalidades generalmente centradas en la asistencia a la escuela y a los controles de salud. Aunque estos elementos son comunes, los PTC difieren significativamente entre países según su concepción, diseño, formas de implementación y arquitectura institucional.

${ }^{3}$ El llamado Consenso de Washington fue impulsado en la región por los organismos de financiación internacional (Fondo Monetario Internacional y Banco Mundial) basado en lógicas neoliberales de estabilidad macroeconómica, crecimiento, liberalización de mercados, atracción de inversiones, reducción del sector público y expansión de los sectores privados. Se impulsaron recortes al gasto social, priorizando la aplicación de programas focalizados y subsidiarios dirigidos a los sectores más pobres. 
de inclusión política (cambios constitucionales legales, reformas electorales, participación política, entre otros aspectos de la consolidación democrática), sino también emergen los debates sobre la inclusión de los temas sociales, referidos tanto a la superación de la pobreza como de la desigualdad.

Las reformas neoliberales fueron comunes en toda América Latina (Barba 2004), persistiendo, en la actualidad, muchos de sus componentes. Estas reformas promovieron cambios estructurales en los planos económico y social, que implicaron el declive del rol del Estado en los países latinoamericanos (Draibe; Riesco, 2007; Martínez Franzoni, 2007). Incluso aquellos países con una vocación redistributiva universalista en sus programas sociales (Argentina, Chile y Uruguay) reorientaron su matriz de bienestar incentivando el aseguramiento individual y el acceso por los propios medios, operando el Estado mediante políticas focalizadas para quienes no pudieran hacer frente a riesgos básicos (Filgueira 2007; Martínez Franzoni, 2007). El gasto social se redujo fuertemente y se llevó a cabo una amplia estrategia de privatizaciones de empresas públicas y de servicios sociales. En ese contexto, las políticas sociales ocuparon un rol subordinado frente a las políticas económicas, y tuvieron un rol paliativo para aquellos sectores de la población sin condicio: nes de participar en el mercado.

¿ quedó fuera de la protección social, forzando \& a los más pobres a depender de la familia, las ô redes sociales y otros mecanismos informales $\overrightarrow{~ d e ~ p r o v i s i o ́ n ~ d e l ~ b i e n e s t a r ~(B a r r i e n t o s ~ 2004 ; ~}$ Martínez Franzoni, 2007). De manera que, desde los propios organismos de financiación, impulsores del Consenso de Washington, se reconoce este fracaso en términos de crecimiento económico y de reducción de la pobreza (Williamson, 2003), críticas hechas también por
Kliksberg (2001), que cuestiona los fracasos del modelo neoliberal basado sólo en lo paliativo y asistencial. Se reorienta así, desde estas instituciones, un giro a las políticas sociales, que van a estar marcadas por el abordaje de la pobreza ya no sólo como subsidios para aumentar ingresos, sino como transferencias monetarias condicionadas a la ampliación del capital humano, evitando la reproducción intergeneracional de la pobreza.

Estos cambios pueden ser mirados desde ángulos distintos. Por una parte, desde diversos autores (Molyneux, 2007; Rawlings, 2004; Serrano, 2005), tras el fracaso de las reformas de ajuste estructural y la incorporación de nuevas prácticas de gestión pública, desde la segunda mitad de los noventa hasta ahora, habría un nuevo modelo de política social 'postneoliberal' que aún está en construcción y que asigna un mayor rol al Estado, impulsado por la expansión de los PTC y otras reformas sociales, aunque no hayan cambios estructurales en el modelo de desarrollo. Molyneux (2007) señala que son reformas de segunda generación, que van más allá del paradigma neoliberal. En tanto, otros (Álvarez, 2005; Estrada Álvarez, 2004; Stolowicz, 2005) plantean que son breves modificaciones en el discurso y la prácticas, pero que es parte de la consolidación del mismo modelo neoliberal ya que no se modifica el principio de subsidiariedad del Estado sobre el mercado. Con la denominada "estrategia pos neoliberal" en que los organismos de financiación internacional traen al Estado de vuelta, el neoliberalismo busca renovarse (ante sus signos de crisis) y presentarse con aires progresistas (Stolowicz, 2005). Coincide Estrada Álvarez (2004) al señalar que estos cambios cumplen una función de legitimación y renovación del neoliberalismo. Además de la búsqueda de gobernabilidad (Stolowicz, 2005).

De manera que, desde la primera visión, lo público y el Estado volvieron a ocupar un rol central, con una nueva generación de políticas sociales que enfatizan la necesidad de univer- 
salizar la protección social y hacer efectivos los derechos sociales (CEPAL 2010; Gomariz 2007; ILO 2011; Molyneux 2007; Ribe; Robalino; Walker, 2010; Sotelsek, 2007). Ha habido una recuperación del rol del Estado, que algunos analistas califican como nueva era de "bienestarismo" y de "esforzándose por conseguir el universalismo” (Molyneux, 2007). Asimismo, Arriagada (2006) sostiene que existe una revalorización del Estado, donde el tema de los derechos sociales cobra un rol relevante. Según señala Gomariz (2007), este giro implica que las políticas sociales hacen un viraje desde el asistencialismo como única estrategia de acción, hacia el encuadre de las políticas en el marco de derechos de los ciudadanos, orientadas a aumentar el bienestar y la cohesión social. Esta orientación implica también una tendencia a la universalización del acceso a la protección, que confluye con el interés de los distintos organismos internacionales de establecer, un cambio desde el predominio en los '90 de la focalización mediante la comprobación de ingresos a un enfoque ampliado más universal en la provisión del bienestar (Deacon, 2005).

Sin embargo, desde la visión crítica a este proceso, se plantea que se continúa con la estrategia de focalización, y el aseguramiento individual de los riesgos, lo que, según Sojo (2003), continuaría contraponiendo las políticas focalizadas a las universales y desestimando la solidaridad en los sistemas de aseguramiento. Por otra parte, el buque insignia de esta estrategia, los PTC, serían, a juicio de Álvarez (2005), un mecanismo de legitimidad de regímenes políticos que han garantizado la consolidación del orden neoliberal en sus países, ya que promueven la potenciación de “activos" de los propios pobres, dándoles valor económico, mientras que el Estado garantiza sólo mínimos biológicos, entregando "paquetes básicos" en áreas que, antes, estaban desmercantilizadas, como la salud y la educación, sin abordar ni resolver los problemas de desigualdad y condiciones estructurales de inequidad. La autora la denomina esta estrategia como focopolítica, ya que el mercado regula la vida de los "capaces", y el Estado, a partir de la gestión y promoción de las "organizaciones de la sociedad civil”, promueve la vida al nivel de mínimos básicos, conteniendo las demandas de los sectores más vulnerables.

Las discusiones en el espectro entre estas posturas aborda la difícil conjunción entre universalidad y focalización, mínimos sociales como derechos y derechos universales, entre capacidades individuales y solidaridad colectiva, entre otras.

Entendiendo que más allá de la discusión sobre los PTC y el rol de los organismos multilaterales, en muchos países, se impulsan revisiones de las políticas sociales y su refundación en un amplio ámbito de iniciativas. En Chile, Brasil, Argentina y Venezuela, hay debates en curso sobre la necesidad de aumentar el alcance de las políticas estatales en muchos ámbitos, como la educación, el alcance de los programas de transferencias monetarias, la ampliación de la negociación colectiva, y extensión de las políticas de salud y educación (Cortés, 2008). Entre los ejemplos de cambios, destaca la reforma de la salud en Uruguay, el plan AUGE (Acceso Universal con Garantías Explícitas) en Chile, el pionero Sistema Único de Salud (SUS) de Brasil, el seguro popular en México y la reforma de la salud en Colombia. También la reforma del sistema de pensiones y las pensiones solidarias en Chile, las pensiones universales no contributivas en el Distrito Federal de México (así como su extensión con variaciones a otros estados mexicanos) y el programa de la Secretaría de Desarrollo Social (SEDESOL) para adultos mayores de 70 años en localidades de hasta 30.000 habitantes, la reforma de jubilaciones en la Argentina y los beneficios de prestación continuada en el Brasil ejemplifican el fortalecimiento de la protección social (CEPAL, 2010). Además, se debe destacar la reciente creación, en Argentina, de la Asignación Universal por Hijo (AUH), que beneficia a trabajadores informales, desocupados y servicio doméstico. Estas son algunas 
muestras de cambios y movimientos que apuntan por lo menos a una inflexión significativa de la ideología neoliberal y sus orientaciones.

En este marco de cambios con diversas velocidades, es interesante analizar las reformas sociales en Chile, pues muestran características específicas que lo convierten en un caso que ilumine los cambios del modelo neoliberal en la región. Chile fue el país de América Latina donde se aplicaron con más intensidad y más prematuramente las recomendaciones de los fondos de financiación internacional, dejando una nueva arquitectura de la protección social con alto grado de privatización de los servicios. Por otra parte, destaca la estabilidad política, con la continuidad de la Coalición Concertación de Partidos por la Democracia, de centroizquierda, que gobernó entre 1990 y 2010. La continuidad de los gobiernos fue un elemento de estabilidad en las reformas sociales emprendidas, que se realizaron con cambios incrementales y sin radicales cambios de políticas y programas al cambiar el gobierno electo. Por otra parte, el cambio democrático implicó la mantención de las bases del modelo neoliberal en lo económico, con una mezcla de énfasis en el gasto social, bajo el lema de 'crecimiento con equidad'. Desde la recuperación de la democracia en 1990, hubo una revitalización de las políticas sociales y se establece ڤ્ un modelo 'híbrido' (Castell, 2005), en que se mantienen las reformas privatizadoras de los

${ }^{4}$ Las privatizaciones de la previsión, la introducción del sector privado en la salud y educación, la internacionalización de la economía, el énfasis en la focalización, los equilibrios macroeconómicos, la liberalización de las relaciones laborales, entre otros, son reformas que prevalecen actualmente en Chile.
Hubo resultados y logros de las políticas públicas implementadas: reducción de más de la mitad de la pobreza (pasó de 38,6\% en 1990 a $14,4 \%$ en 2011), amplio acceso y cobertura de bienes y servicios sociales, y altos niveles de desarrollo humano (IDH de 0,805 en 2011). Sin embargo, la promesa de equidad no fue realizada (en 2011, el Gini fue de 0,52 casi sin variación desde 1990). Chile es uno de los países con mayores niveles de desigualdad en el mundo, que se refleja no sólo en inequidades en el ingreso, sino también en la calidad de los servicios, cumpliéndose la premisa de 'pobres servicios para los pobres' (Martner, 2010). Finalmente, destacan las reformas sociales, en el ámbito de la educación (1996), de la creación del seguro de desempleo (2002) y su extensión (2009), la creación del Sistema de Protección Social Chile Solidario (2004), la reforma de la salud AUGE (2004), la creación del Sistema de Protección a la Infancia Chile Crece Contigo (2006) y la Reforma Previsional (2008), que buscaron superar las falencias del modelo de crecimiento con equidad (Martin, 2009). Estos déficits del modelo de crecimiento con equidad se empiezan a mostrar con mayor claridad a inicios de los 2000, y la agenda de reformas sociales se impulsa con el cambio en la Presidencia con un socialista a la cabeza (Ricardo Lagos). Luego vendrá el gobierno de la también socialista Michelle Bachelet, marcando ambos importantes reformas en el ámbito social e instalando el Sistema de Protección social basado en derechos.

De este modo, se pueden establecer, entre 1990 y 2010, dos períodos en políticas sociales. El primero (1990-2000) marcado por la equidad, gobernabilidad y desarrollo en democracia y el segundo (2000-2010) con mayor centralidad de las políticas de igualdad y protección de derechos sociales. En el primer período, el Estado asume una función social activa que se traduce en un aumento del gasto social. La política social prioritariamente, se hizo cargo de la pobreza que afectaba a más del cuarenta por ciento de los chilenos. Sólo en los últimos años se avanza hacia combinar la focalización con una mayor ampliación universal a 
ciertos beneficios y derechos garantizados. En el segundo, se cambió la orientación de las políticas sociales, al pasar de un enfoque basado en las necesidades a otro basado en el derecho a la protección social, garantizando derechos sociales. Progresivamente, se buscó universalizar el acceso a servicios en las áreas de salud, pensión, desempleo, cuidado infantil y educación primaria. Desde un enfoque residual y temporal, se pasó a constituir una protección social con perspectiva de piso, convirtiendo a la protección social en un componente permanente de la estrategia de desarrollo para el crecimiento inclusivo (ILO, 2011).

De las reformas sociales emblemáticas de ese período (2000-2010), este estudio abarca la creación del Sistema de Protección Social Chile Solidario, en el gobierno de Lagos (2000-2006), y la Reforma Previsional, durante el gobierno de Bachelet (2006-2010). Las razones de la elección se fundan en que son reformas emblemáticas por su grado de innovación, impacto en otras políticas y extensión de sus beneficios. Chile Solidario es la reforma más sustantiva realizada hasta la fecha en el componente asistencial o no contributivo de la protección social. La Reforma Previsional, a su vez, es la reforma paradigmática del componente de seguridad social, en un sistema de pensiones completamente privado, como es el caso chileno. Chile Solidario generó un punto de partida para el proceso de cambio de las políticas sociales. Desde una visión centrada en el componente asistencial y caracterizado por políticas sectoriales, se pasó a la búsqueda de políticas y programas que incluyeran un modelo de gestión integrado, multisectorial y que incorporara una noción multidimensional de la pobreza. Este proceso implicó una transformación relevante en términos de constituirse como una política innovadora de superación de la pobreza, que enfatizaba el desarrollo de capacidades junto con el mejoramiento de ingresos (Palma; Urzúa 2005; Raczynski 2008; Villatoro 2007). Por otra parte, la Reforma Previsional mantuvo la provisión privada del sistema de pensiones, pero introdujo un pilar solidario, no contributivo y solidario, con énfasis en la incorporación de elementos redistributivos (Martner 2010). También se generaron mayores regulaciones. El rol del Estado en previsión social se fortaleció con la incorporación de la noción de derechos sociales y la vinculación de las políticas sociales con la ciudadanía (Délano, 2010; Arenas de Mesa, 2010). Así, estas dos reformas buscaron asentar las bases de un Sistema de Protección Social basado en derechos, que ampliara de manera progresiva la cobertura de las prestaciones, cubriendo los riesgos a lo largo del ciclo vital.

Los desafíos que abre el caso chileno se vinculan a los que se establecen en el contexto de América Latina. Al igual que muchos países de la región, Chile pareciera estar en un momento de toma de decisiones sobre alternativas de políticas públicas con respecto a los cambios requeridos en la protección social, cuya ecuación no está clara en el sentido de si planteará políticas que hagan frente a la desigualdad y la pobreza, en un marco en que confluyan crecimiento económico, desarrollo social y profundización democrática. Hay un creciente consenso en la región sobre la necesidad de reformar la desigual distribución del ingreso y la inclusión social, y la reforma de la protección social podría contribuir al logro de esos objetivos. Sin embargo, la reorientación de la intervención social del Estado lleva tiempo, y su resultado dependerá de las posibilidades de llegar a un consenso entre los diferentes actores institucionales, incluidos los partidos políticos, sindicatos y las organizaciones sociales (CEPAL 2010; Cortés 2008).

Por tanto, no están claros los caminos que tomarán estas reformas, ni la cobertura y extensión que alcanzarán las prestaciones. Tampoco si habrá cambios incrementales o reformas profundas que cambien completamente lo obrado por las reformas neoliberales. Se vislumbran grietas importantes mientras que actores y fuerzas muy poderosas internas y externas actúan en el trasfondo, impulsando 
cambios de rumbo (Cimoli et al., 2005). En este marco, Chile se encuentra, como señalan Cecchini y Martínez (2011), en el grupo de países de América Latina que tienen una impronta y tradición de enfoque de derechos en sus políticas sociales. Donde, como señalan Draibe y Riesco (2007), pese a los cambios neoliberales, el sustrato de cobertura universal de la salud y educación mantuvo, como resultado de la path dependency. ${ }^{5}$

En este punto se sitúa la pregunta central de este estudio, en cuanto es relevante estudiar este quiebre en la experiencia chilena como un caso particular desde una mirada de más largo plazo. Los procesos señalados parecen constituir manifestaciones de un momento complejo, en el cual buscan desenvolverse tensiones que cruzan diversos planos y atraviesan diferentes dimensiones. Por una parte, parece coincidir con un momento de inflexión en el modelo social y la estrategia general de desarrollo del país, en un contexto que trasciende al nivel regional. Tiene todas las trazas de constituir el trasfondo general del momento, y por lo tanto, el criterio principal a considerar en el momento de determinar el curso a seguir.

Este cambio constituye un proceso significativo en términos del modelo de protección social, (Draibe; Riesco 2007), en el sentido que existe una inflexión (no un fin) en el ثิ modelo neoliberal y sus orientaciones, en la lógica de fortalecimiento de las políticas sociales, surgiendo un nuevo Estado desarrollista del bienestar, siendo relevante de investigar, en cuanto existe un debate sobre los límites $\rightarrow$ y posibilidades de esta transformación y de os la introducción del enfoque de derechos en ¿ la protección social en Chile (Martner, 2010; iे Raczynski, 2008). Asimismo, la estrategia $\vec{s}$ emergente sugiere una nueva relación entre lo ฮั)

స్ ${ }^{5}$ La teoría de path dependency establece que las decisiones están condicionadas por la trayectoria (procedimien-

T.

cisiones tomadas en el pasado. El cambio de las políticas

- públicas será de corte evolutivo y progresivo. Así habría

ocurrido en el caso chileno, en que subsistieron políticas

universales de salud y educación, pese a la impronta neo-

liberal impuesta en dictadura. la inclusión, las políticas sociales universales y la vinculación Estado ciudadanía. Aunque la noción de derechos pasa a estar en el centro de la formulación del Sistema de Protección Social, se observa el desarrollo, aún en construcción, de un enfoque de protección como garantía ciudadana (Cecchini; Martínez, 2011; Raczynski, 2008).

Por otra parte, también es central, en la definición de este estudio, analizar el cambio en las orientaciones de las políticas de protección social, utilizando las herramientas analíticas que provee el análisis de "marcos interpretativos", como métodos de estudio en políticas públicas que permiten poner foco en las alternativas ideológicas, de valores, las ideas que están a la base de la definición de los problemas que impulsan el cambio en las políticas y las alternativas que se plantean. Lo que resulta especialmente relevante en casos, como es el chileno, según hemos señalado, en donde se despliegan cambios importantes, habiendo poca claridad sobre cómo, con qué disputas ideológicas y valóricas, desde el campo político, se han configurado los problemas (en este caso la protección social). Y en donde interesa analizar si, efectivamente, las reformas han implicado un punto de inflexión o cuánta profundidad han alcanzado respecto del cambio en un contexto particular. Ello es central como foco de relevancia de este estudio, en que se aborda la problemática investigada desde el enfoque constructivista de las políticas públicas, utilizándolo en virtud de que permite develar las orientaciones ideológicas, políticas o valóricas que sostienen los actores que participan en la definición de los problemas y que impactan en la formulación de las políticas. En este plano analítico, hay una contribución de la perspectiva constructivista, que es clave para analizar la significación de los problemas públicos y la orientación de cambio de políticas que, sin embargo, no han sido puestas a prueba, siendo necesario avanzar en ese campo.

Es relevante analizar y estudiar el cambio de políticas desde esta perspectiva, pues los 
análisis centrados en las ideas, como sostienen Sabatier y Schlager (2000), Schmidt y Radaelli (2004), Muller (2000), Forester (1993) y Fischer (2003), muestran un mayor interés en estudiar el cambio como un factor central en el análisis de políticas públicas. En tanto modelo racionalista, predominante en la actualidad, tiene límites a ese respecto, en cuanto está centrado en los intereses individuales, basado en un modelo que no permite poner en estudio cómo los sistemas de significados y los marcos interpretativos impactan en la forma en que los actores perciben y modifican sus intereses, y desarrollan construcciones conjuntas al interpretar la realidad (Fischer; 2003). Así también, por otra parte, el análisis de políticas públicas desde esta perspectiva permite, según señalan Forester, (1993), De León (2006) y Hajer (2003), legitimar una mayor participación de los ciudadanos para afianzar la democratización de los procesos de políticas públicas. En cuanto, desde esta perspectiva analítica y metodológica constructivista, se entiende que las políticas son procesos de creación y control de sistemas compartidos de significado social respecto de los problemas, así como de las soluciones, en donde resultan esenciales las dinámicas de competición, negociación y diálogo de significados e interpretaciones. De forma tal, que las dimensiones intangibles o simbólicas del proceso de políticas públicas adquieren centralidad en las dinámicas que dan cuenta de la estabilidad y del cambio de una política. ${ }^{6}$

Complementariamente es relevante destacar que esta perspectiva de análisis contribuye y hace parte de la evolución reciente en materia de análisis de política públicas, ocurrida, como señalan Howlett y Cashore (2007), entre una 'primera ortodoxia', centrada en las lógicas incrementales o evolucionarias de las políticas públicas, hasta llegar a la 'ortodoxia actual', donde priman los análisis no centrados en el

${ }^{6}$ Las políticas públicas pueden analizarse desde su dimensión simbólica (los discursos, principios, ideas y valores), desde su dimensión sustantiva (o de contenido, de las necesidades que cubren, intensidad y cobertura), y desde la dimensión operativa (de los instrumentos para la intervención formas de provisión, organización y gestión). modelo de etapas, que se desarrollan desde la vertiente pos positivista, enfatizando el rol de las ideas en el desarrollo y cambio de las políticas públicas. Estos últimos, según sostienen Sabatier y Schlager (2000), Schmidt y Radaelli (2004), Muller (2000), Forester (1993) y Fischer (2003) muestran un mayor interés en estudiar el cambio como un factor central en el análisis de políticas, poniendo foco, como recogemos de Fischer (2003), en los sistemas de significado, los marcos interpretativos que impactan en la forma en que los actores perciben y modifican sus intereses y desarrollan construcciones conjuntas al interpretar la realidad.

De esta manera, en el marco de los dos marcos de antecedentes expuestos, el problema de estudio de esta investigación queda definido por la necesidad de explorar y poner en uso y prueba el análisis del cambio propuesto por la perspectiva constructivista en el estudio de políticas públicas, analizando los marcos interpretativos, específicamente los "policy frames” que están en juego en la definición de problemas y soluciones en los desarrollos y orientación de las políticas sociales y su vinculación con la construcción de Sistemas de Protección Social, en el caso chileno, concibiendo a estos como una producción relacional, que integra dinámicas de negociación y conflicto de actores, aportando tanto a la comprensión del cambio en los sistemas de protección social en Chile y en la Región. A la vez que poniendo en uso los recursos metodológicos que provee el "policy frames analysis", en cuanto modelo conceptual y analítico que no ha sido utilizado para analizar procesos de cambio de políticas en el campo de las políticas sociales en América Latina, altamente dominado por el análisis del ciclo de políticas y el racionalismo económico.

Partiendo del análisis de las reformas que están en curso en materia de protección social en Chile, se analiza su fase de formulación, guiados por la pregunta central de investigación: ¿Cuáles son las concepciones explícitas e implícitas de los actores sociopolíticos sobre los 
problemas y soluciones de la protección social en Chile, que permiten comprender el proceso y la dirección del cambio del Sistema de Protección Social? Buscando responder las preguntas específicas de: ¿Cuál es el problema tal y cómo se representa?, así como ¿Qué solución se ofrece al problema?, y ¿Cuáles son los marcos interpretativos principales, complementarios y alternativos, relacionados con el cambio en el Sistema de Protección Social chileno?

El objetivo general definido es analizar la dimensión simbólica de las políticas de protección social, estableciendo el conflicto de marcos interpretativos de las políticas sociales que permiten comprender el cambio y continuidad del Sistema de Protección Social en Chile (2000 a 2010), y determinando las comunidades de interpretación presentes en los casos de Chile Solidario y Reforma Previsional. Así como, a nivel de objetivos específicos, se define: (1) establecer los Marcos Interpretativos principales, secundarios y alternativos desde los cuales se define cada etapa de desarrollo de los Sistemas de Protección Social chilenos en el período de estudio; (2) determinar y caracterizar las comunidades de interpretación sobre protección social, estableciendo cuáles son los objetivos de las acciones de protección social, los problemas a los cuales refieren y las soluciones que buscan; (3) analizar ثิ los componentes de los conflictos de marcos interpretativos sostenidos en torno a la construcción del Sistema de Protección Social en Chile que explican la orientación del cambio.

\section{ENFOQUE METODOLÓGICO}

El marco metodológico base del estudio es la Teoría Fundamentada (Strauss; Corbin, 2002). Se escogió tanto por su potencialidad explicativa y fortaleza en la construcción de teoría que aporte al objeto de estudio, como por su epistemología constructivista, que muestra gran afinidad con el marco teórico escogido, de análisis de marcos interpretativos de las políticas. Como estrategia investigativa complementaria, se utilizó el Estudio de Casos (Yin, 2004), que tiene, entre sus características principales, el ser un enfoque investigativo cualitativo, que vincula el caso de estudio con su contexto y viceversa.

Combinaciones similares entre el enfoque de investigación cualitativa de Estudios de Casos y la metodología de la Teoría Fundamentada se han realizado en diversos campos de las ciencias sociales, con resultados muy efectivos (Stern, 2007). La Teoría Fundamentada tiene precisamente como fin el construir teoría a enraizada en los datos producidos inductivamente (Charmaz, 2006; Strauss; Corbin, 2002), utilizando herramientas sistemáticas para alcanzar abstracciones de valor teórico, sin sacrificar la complejidad, permitiendo un análisis dinámico e interactivo, con conclusiones que muestran relaciones en un amplio campo de influencias (Stern, 2007). El Estudio de Casos permite acceder a la complejidad de las relaciones en casos específicos, sin separar el caso de su contexto. Y, como forma de investigación, busca contribuir a la construcción y desarrollo de perspectivas teóricas (Yin, 2004). El diseño metodológico corresponde al de múltiples casos, diseño incrustado (Yin, 2004), en que se analizan dos o más casos con dos o más unidades de análisis. Con ello se busca la replicación lógica de los resultados repitiendo el mismo estudio sobre casos diferentes para obtener más pruebas y mejorar la validez externa de la investigación. Se analizan, como se aprecia en la Cuadro 1, dos casos de cambio sustantivo en protección social: 1) Chile Solidario, del componente seguridad social del Sistema de Protección Social, del gobierno de Lagos (2000-2006); y 2) la Reforma Previsional como componente de la seguridad social del gobierno de Bachelet (2006-2010).

Las unidades de análisis están determinadas por: establecimiento de marcos sobre la protección social en ambos casos de estudio; la constitución de comunidades interpretativas; el establecimiento del conflicto de marcos; y la elaboración de relaciones explicativas so- 


\begin{tabular}{|c|c|c|}
\hline & \multicolumn{2}{|c|}{ Pilares de la protección social } \\
\hline & Seguridad social & Asistencia social \\
\hline Objetivos & $\begin{array}{l}\text { - Programas proveen protección a lo largo ciclo vital } \\
\text { - Protegen frente a maternidad, vejez o contingencias } \\
\text { el empleo (accidentes, discapacidad) } \\
\text { - Financiada con aportes mixtos (estado, trabajadores, } \\
\text { empleadores) }\end{array}$ & $\begin{array}{l}\text { - Provee apoyo a quienes están } \\
\text { en pobreza } \\
\text { - Financiada con impuestos }\end{array}$ \\
\hline Casos seleccionados & Reforma previsional & $\begin{array}{l}\text { Sistema de Protección Social } \\
\text { Chile Solidario }\end{array}$ \\
\hline Gobierno & Gobierno de Bachelet (2006-2010) & $\begin{array}{l}\text { Gobierno de Lagos } \\
(2000-2006)\end{array}$ \\
\hline Coalición & Concertación de Partidos por la Democracia & $\begin{array}{l}\text { Concertación de Partidos por } \\
\text { la Democracia }\end{array}$ \\
\hline Partido Presidente & Partido Socialista & $\begin{array}{l}\text { Partido Por la Democracia - } \\
\text { Partido Sociali sta }\end{array}$ \\
\hline $\begin{array}{l}\text { Discusión } \\
\text { parlamentaria }\end{array}$ & $\begin{array}{l}\text { SI } \\
\text { Ley } 19.949 \text { ( } 5 \text { de junio 2004) }\end{array}$ & $\begin{array}{l}\text { SI } \\
\text { Ley } 20.255 \text { ( } 17 \text { marzo 2008) } \\
\text { Ley } 20.233 \text { (22 julio 2009) }\end{array}$ \\
\hline
\end{tabular}

Fonte: Elaboración Propia.

bre la interpretación de la protección social. Respecto de las fuentes de información, en un inicio, se seleccionaron las discusiones parlamentarias de las dos grandes instancias de cambio en materia de seguridad social y asistencia en la protección social. A medida que se avanzó en el análisis de categorías, y siguiendo los procedimientos de selección de la Teoría Fundamentada, se optó por incorporar nuevos elementos que servirían para responder a las preguntas de investigación que iban surgiendo o desarrollándose en el análisis. Así se analizaron los documentos emanados por el conjunto de Think Tanks, y también los estudios y documentos provenientes de la discusión de la Comisión Presidencial de Reforma Previsional, así como los documentos aportados por la sociedad civil y academia al debate parlamentario. Estos datos fueron el material documental base de la política social en análisis, los cuales fueron entendidos como textos dotados de una coherencia narrativa que organiza y estructura la realidad, que permite la comprensión y explicación fiable y contextualizada de la producción y negociación de una política pública (su definición, su diseño e implementación) y de las dinámicas de las comunidades interpre- tativas, actores sociales (técnicos y políticos), implicada como dimensión constituida y constituyente de estos marcos de política (Schön; Rein, 1994; Verloo, 2002).

El análisis de los datos se realizó conforme al Método Comparativo Constante, propuesto por la Teoría Fundamentada (Charmaz, 2006; Strauss; Corbin, 2002), que define la globalidad del procedimiento analítico de este enfoque metodológico. Consiste en someter a un análisis comparativo y sistemático cada uno de los incidentes contenidos en los datos que resulten relevantes al objetivo o pregunta de estudio, combinando, para ello, los procedimiento o niveles de codificación: Codificación Abierta, Codificación Axial y Codificación Selectiva (Charmaz, 2006; Strauss; Corbin 2002), con el objetivo de, por una parte, generar categorías de datos (definiendo, de manera densa, sus propiedades y dimensiones), y relaciones entre ellas, basándose en la detección de patrones de comportamientos repetidos y regulares en los datos. Por otra, tiene el objetivo de generar teorías pertinentes, contextualizadas y corroboradas en los datos producidos, siguiendo, para ello, el sistema o procedimiento de muestreo teórico (Trinidad, Carrero; Soriano 2006). 


\section{RESULTADOS}

Como se observa en lo Cuadro 2, desde el análisis integrativo de los frames presentes tanto en Chile Solidario como en la Reforma Previsional, se establece que los policy frames distinguidos no ocupan igual posición respecto de la implementación concreta del programa, de manera tal que es posible distinguir un policy frames que se aproxima y a los énfasis que destacan en el programa concretamente implementado, que llamaremos policy frames principal (MIH). Así también se observa que hay un tipo de marco interpretativo que juega un rol antagónico y de tensión principal con el marco interpretativo principal. Este marco interpretativo no principal es el que crea la polaridad, pues, ante él, éste debate, discute, negocia. A su vez, influye como eje de presión significativo al marco interpretativo principal, siendo posible asumir que estos marcos interpretativos son parte fundamental del proceso de construcción y delimitación del marco interpretativo principal. Aunque no guían cercanamente la concreción de los programas estudiados, sí mantienen como importante influencia y establecen límites y condiciones para la concreción final del que será el frame principal. De esta manera, lo llamaremos Marco Interpretativo Complementario (PFC). A la vez que se distingue un tercer claramente perfilado que, aunque aparece y ejerce una posición de

Cuadro 2 - Codificación selectiva integrada Chile solidario y reforma previsional

\begin{tabular}{|c|c|c|c|}
\hline $\begin{array}{l}\text { Marco } \\
\text { interpretativo }\end{array}$ & $\begin{array}{l}\text { Sistema Protección Social } \\
\text { Chile Solidario }\end{array}$ & Reforma Previsional & $\begin{array}{l}\text { Meta análisis Chile } \\
\text { Solidario más } \\
\text { reforma previsional }\end{array}$ \\
\hline $\begin{array}{l}\text { Principal } \\
\text { Axial }\end{array}$ & $\begin{array}{l}\text { Sistema de Protección Social } \\
\text { integrado multidimensional de } \\
\text { aseguramiento de mínimos y } \\
\text { desarrollo de capacidades como } \\
\text { protección garantizada por } \\
\text { derechos como vía de superación } \\
\text { de la extrema pobreza. }\end{array}$ & $\begin{array}{l}\text { Cambio del sistema previsional } \\
\text { con corrección de la inequidad } \\
\text { generada por la capitalización } \\
\text { individual mediante protección } \\
\text { social de mínimos previsionales } \\
\text { garantizados según derecho y } \\
\text { fortalecimiento de la regulación } \\
\text { estatal de la industria previsional. }\end{array}$ & $\begin{array}{l}\text { Aseguramiento de } \\
\text { mínimos, como } \\
\text { derechos mínimos } \\
\text { como acción estatal } \\
\text { de protección social }\end{array}$ \\
\hline $\begin{array}{l}\text { Secundario } \\
\text { Axial }\end{array}$ & $\begin{array}{l}\text { Sistema de protección social de } \\
\text { derechos sociales universales con } \\
\text { más Estado. }\end{array}$ & $\begin{array}{l}\text { Sistema previsional de derechos } \\
\text { sociales universales de efecto } \\
\text { redistributivo no asistencial. }\end{array}$ & $\begin{array}{l}\text { Protección social con } \\
\text { derechos sociales } \\
\text { universales y de efecto } \\
\text { redistributivo como } \\
\text { política social no } \\
\text { asistencial. }\end{array}$ \\
\hline $\begin{array}{l}\text { Complementario } \\
\text { Axial }\end{array}$ & $\begin{array}{l}\text { Menos Estado, más crecimiento } \\
\text { económico, más descentralización } \\
\text { y más focalización como vía de } \\
\text { superación de la extrema pobreza }\end{array}$ & $\begin{array}{l}\text { Subsanar imperfecciones de la } \\
\text { capitalización individual, } \\
\text { aumentar el crecimiento } \\
\text { económico manteniendo rol } \\
\text { subsidiario del Estado Subsidiario }\end{array}$ & $\begin{array}{l}\text { Crecimiento } \\
\text { Económico y Estado } \\
\text { Subsidiario (menos } \\
\text { Estado) como } \\
\text { respuesta a las } \\
\text { necesidades sociales } \\
\text { (problemas sociales) }\end{array}$ \\
\hline $\begin{array}{l}\text { Conflicto } \\
\text { de marcos } \\
\text { interpretativos }\end{array}$ & $\begin{array}{l}\text { Piso mínimo de protección social } \\
\text { regulación y fiscalización del } \\
\text { mercado como funciones del } \\
\text { Estado versus política social como } \\
\text { crecimiento económico y función } \\
\text { de asistencia del Estado en un }\end{array}$ & $\begin{array}{l}\text { Derechos sociales como } \\
\text { mínimos garantizados por el } \\
\text { Estado versus derechos } \\
\text { sociales como protección } \\
\text { social universal con efecto } \\
\text { redistributivo }\end{array}$ & Categoría selectiva \\
\hline
\end{tabular}

marco de subsidiaridad.

\section{CONFLICTO ENTRE BIENESTARISMO Y NEOLIBERALISMO}

En el contexto de los Marcos Interpretativos Principal Meta y Complementarios Meta, la categoría que permite explicar o entender el cambio en el Sistema de Protección Social en Chile es el proceso de conflicto, tensión y negociación entre bienestarismo y neoliberalismo en la concepción. 
referencia que se vincula y debate tanto con los marcos complementario y principal, no tiene presencia directa y se ubica distante de la concreción del programa en análisis, y, por tanto, es posible atribuir que posee menos fuerza de influencia y de visibilización, que llamaremos marco interpretativo secundario (PFS).

En segundo lugar, desde la observación conjunta e integrada de cada uno de los marcos interpretativos que se observan en cada uno de los dos programas analizados, se realiza un análisis meta, en el cual se deriva la concepción latente y de nivel de abstracción superior que es común a cada marco interpretativo, tanto de Chile Solidario como de la Reforma Previsional. Desde este análisis se produce, por tanto, un marco interpretativo Principal Meta (PFHM), así como un marco interpretativo Complementario Meta (PFCM), y un marco interpretativo Secundario Meta (PFSM), a la vez que, desde el análisis conjunto de estos, se derivan los Conflictos de Frames Meta (PFM).

Si consideramos los componentes principales en el Marco Interpretativo Principal de Chile Solidario, se puede observar que aparecen características tales como: que es un sistema integrado e integral, es decir, de carácter multidimensional, que involucra el aseguramiento de mínimos y el desarrollo de capacidades así como la protección garantizada de ciertos beneficios como vía de superación de la extrema pobreza. A su vez, el Marco Interpretativo Principal de la Reforma Previsional establece como componentes principales que es necesario un cambio del sistema previsional a través de la corrección de la inequidad que genera el mecanismo de capitalización individual por medio desarrollo de un componente de protección social, orientado al aseguramiento y garantía de mínimos previsionales según derechos para fortalecimiento de la regulación estatal del mercado de la industria previsional.

Podemos derivar que el marco interpretativo común, que integra las visiones tanto de Chile Solidario como de la Reforma Previsio- nal es el marco interpretativo Principal Meta, que refiere al Aseguramiento de mínimos como derechos como piso de la acción estatal de protección social. Es decir que pone, como centro de la acción estatal, la protección social que garantiza derechos mínimos o piso de protección social. A su vez, podemos establecer un Marco Interpretativo complementario meta (PFCM), que integra los frames complementarios tanto de Chile Solidario como de la Reforma Previsional. Respecto del Marco Interpretativo Complementario de Chile Solidario, este puede resumirse como un rol central del crecimiento económico y una menor necesidad de injerencia del Estado (en una lógica subsidiaria), más descentralización y focalización como vía de superación de la extrema pobreza. En tanto, el Marco Interpretativo Complementario de la Reforma Previsional refiere al perfeccionamiento de las fallas de la capitalización individual, crecimiento económico y Estado subsidiario como bases de los cambios necesarios para el sistema previsional. A la vez, podemos integrar un segundo Marco Interpretativo complementario meta PFCM, que refiere al rol fundamental del crecimiento económico, resumido como: Crecimiento Económico y Estado subsidiario (menos Estado) como respuesta a las necesidades sociales (problemas sociales). A la vez, si observamos el Marco Interpretativo Secundario (PFS) al igual que en los análisis anteriores, se establece un Marco Interpretativos Secundario Meta (PFSM), particularmente en la Reforma Previsional, que refiere a un sistema previsional de derechos social, universal, de efecto redistributivo y no asistencial. Derivamos un PFSM que refiere a Derechos Sociales Universales y de efecto redistributivo como política social no asistencial. Ahora bien, si observamos los conflictos de frames (CF) en los dos programas analizados, podemos analíticamente derivar los conflictos de frames meta (CFM) en el mismo sentido de los análisis anteriores.

Así, vemos los conflictos de frames del programa Chile Solidario referidos a: 
1. Desarrollo de una respuesta sistémica institucional centralizada frente a la pobreza versus una respuesta local, descentralizada y focalizada frente a la pobreza.

2. Respuesta estatal de protección con asistencia, promoción, y sistémica (en red), orientada a aseguramiento de mínimos (integral) versus respuesta focalizada en el mejoramiento de los ingresos en grupos específicos que presentan carencia socioeconómica (asistencia focalizada) como un paliativo ante fases en que el desarrollo económico no logra satisfacer estas necesidades en el conjunto de la población.

3. Crecimiento económico, más inversión social contracíclica versus crecimiento económico y políticas sociales anticíclicas.

4. Pobreza extrema como un problema multidimensional (oportunidades, capacidades y promoción) de carácter sociocultural versus pobreza extrema como un problema socio económico relativo al nivel de ingresos.

5. Función de protección del Estado versus Estado subsidiario.

6. Condiciones mínimas garantizados por el Estado en virtud de derechos versus aseguramiento de asistencia focalizada discrecional.

Y los conflictos de frames de la Reforma Previsional están referidos a:

1. Sistema de capitalización como un sistema inequitativo, que requiere regulación desde el : Estado para asegurar derechos previsionales mínimos versus sistema de capitalización individual como un sistema que funciona adecuadamente y requiere ajustes específicos en el funcionamiento del mercado, en su cobertura para determinados grupos y sus mejora depende del crecimiento económico.

2. Necesidad de regulación y fiscalización desi de el Estado y la ciudadanía de la industria previsional versus libre competencia del mercado de administración previsional y subsidiaridad del Estado frente a él.

3. Participación de Estado en la industria previsional como forma de regular la industria previsional y el mercado de capitales en favor de los intereses de los trabajadores y del país versus participación del Estado en la industria previsional como riesgo de pérdida de autonomía de la gestión de ésta ante las presiones políticas y corporativas y la corrupción.

4. Pilar Solidario como garantía estatal del derecho a mínimos previsionales versus Pilar Solidario como un mejoramiento y ampliación de las pensiones asistenciales logrado por el crecimiento económico del país.

5. Reforma Previsional como reconocimiento de un derecho social de mínimo previsional versus Reforma Asistencial como un sistema que no reconoce derechos, que genera pensiones asistenciales (Pilar Solidario) y mantiene un mecanismo (Capitalización Individual) individualista no redistributivo que mantiene desigualdad.

Podemos analíticamente derivar dos conflictos de frames meta, es decir que cruzan a ambos programas. El primero referido a política social como protección social por aseguramiento de mínimos y regulación y fiscalización del mercado como funciones del Estado versus política social como crecimiento económico y función de asistencia del Estado en un marco de subsidiaridad de éste. El segundo referido a los derechos sociales como mínimos garantizados por el Estado versus derechos sociales como protección social universal con efecto redistributivo.

En el análisis selectivo que presentamos en el Cuadro 2, establecemos que la categoría principal, en el contexto de estos Marcos Interpretativos Meta y de los conflictos de frames Meta, la categoría que permite explicar o entender el cambio en el Sistema de Protección Social en Chile es el proceso de conflicto, tensión y negociación entre bienestarismo y neoliberalismo, como dos concepciones de políticas públicas de protección social que reflejan la dinámica de constitución del sistema de protección social y las negociaciones y debates que lo cruzan en Chile. A partir de estos conflictos de frames es que se produce el cambio en las políticas de protección social y donde ocurren los fenómenos deliberativos que ge- 
neran posiciones de negociación, en que, al no y que son el fundamento de significados, de vaexistir hegemonía, hay una orientación de la lores, de argumentos y de categorías técnicas de política de protección que es progresiva, que se los problemas y soluciones que se configuran y encamina o ubica cerca del polo 'bienestarista', de las racionalidades técnicas que se observan, sin lograr centrarse completamente en él. tanto en análisis de cada programa como en el

Como se aprecia en la Figura 1, los senti- análisis meta que se acaba de presentar, refieren dos, las distinciones básicas que están en juego de formas y proximidades distintas a posicio-

Figura 1 - Conflicto de FRAMES Y cambio en políticas de protección social

\section{I_Análisis Selectivo Sistema de Protección Social Chile 2000-2010 I}

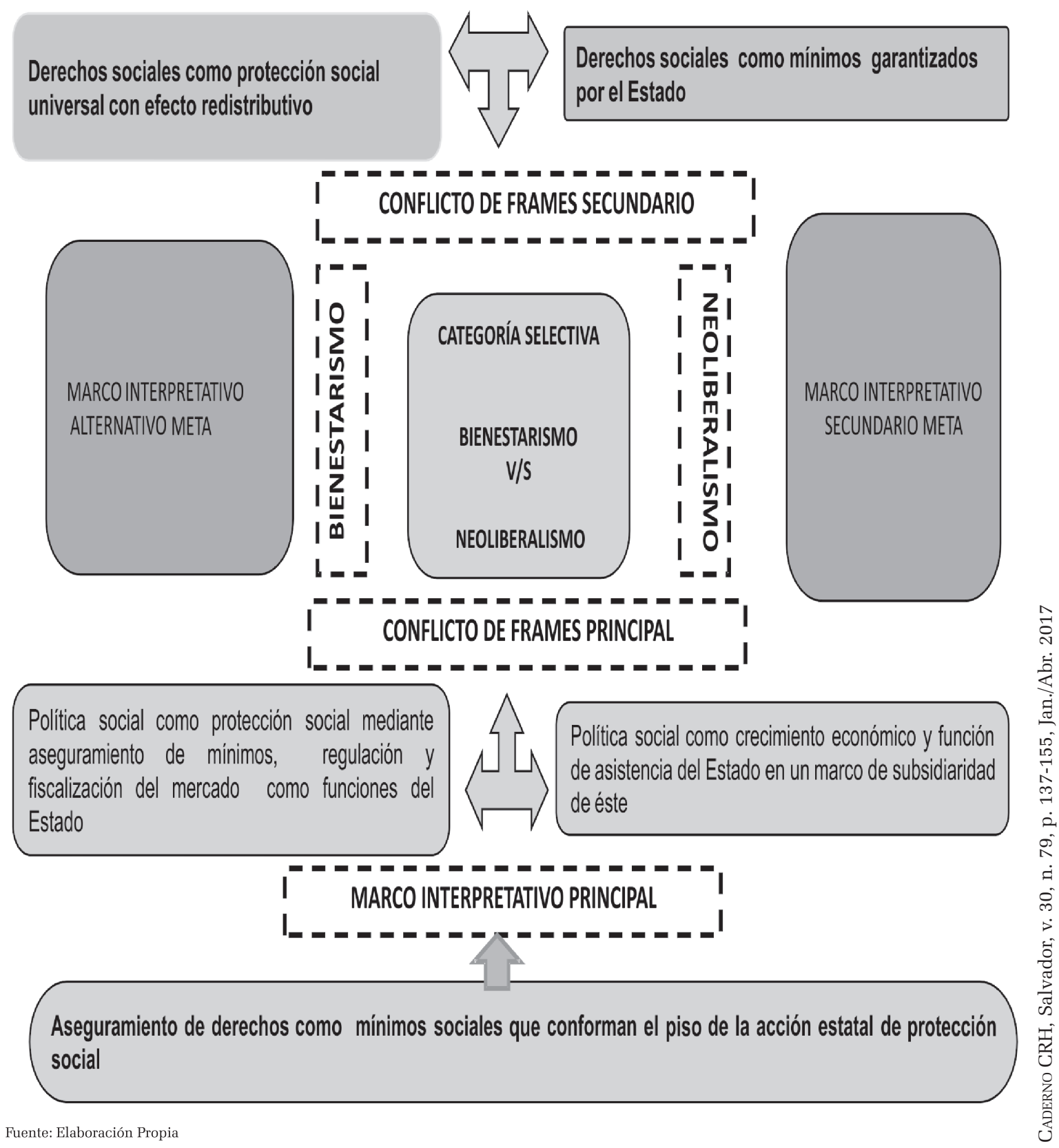


nes ubicadas en el continuo de debate entre el bienestarismo y el neo liberalismo en materia de políticas de protección social.

Las posiciones desarrolladas tanto por los distintos actores y articuladas en los diferentes marcos interpretativos desarrollados por las distintas comunidades interpretativas, así como relativas a las conflictos de frames distinguidos en el análisis, tanto del programa Chile Solidario como en la Reforma Previsional, refieren a uno de estos polos y(o) se ubican en posiciones de cercanía o distancia relativa a éstas. De esta manera, la forma que adquiere el Sistema de Protección Social, según se observa en los programas o reformas estudiadas, se comprende en su estructura general, así como en sus características y mecanismos particulares, como una lucha o tensión entre estas dos orientaciones (bienestarismo y neoliberalismo en políticas públicas) ocurridas en relación a la dinámica de cambio y continuidad de las políticas de protección social

Así, ésta sería la tensión fundamental que mueve o que está en el núcleo del debate o de la lucha política entre comunidades interpretativas que se despliegan en la construcción del sistema de protección social en Chile. Ello nos permite explicar que, en el caso particular de las reformas estudiadas en Chile, ha ocurrido un proceso de conflicto de frames, จे que lleva a procesos de negociación, que impacta por una parte en una implementación de los cambios, en que la implementación de derechos queda como una categoría difusa, poco clara y con garantías acotadas. De este modo, cuando se genera garantía de derechos (como $\dot{\alpha}$ en el caso de la Reforma Previsional), se lo hace desde una lógica focalizada en pobreza, के y cuando se establecen mínimos en Chile Soli-

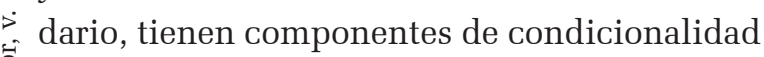
y son mínimos muy básicos.

Respecto del análisis de la dinámica de desarrollo y cambio del Sistema de Protección Social en Chile, las posiciones que se despliegan, tanto las hegemónicas (las que han guiado la implementación), como las complementa- rias o alternativas (las que presionan, limitan e influyen sustancialmente en las ocurrencias), o las secundarias (que aparecen marginalmente o bien son invisibilizadas, aunque influyen vía referencia), así como los conflictos entre los componentes y las distinciones de los marcos de interpretativos que dan base a cada una de estas, se relacionan y se derivan de esta tensión principal.

Desde cada marco interpretativo, la configuración de problemas y soluciones que concurren a ellos, o que son construidos desde ellos, así como las construcciones de sujetos $\mathrm{y}$ argumentaciones que se distinguen, tienen una determinada distancia o bien proximidad respecto de los polos de esta categoría de tensión central y también complementariamente toman posición o refieren y(o) contra argumentan el polo opuesto de esta tensión, respecto de donde se ubican.

Por tanto, de alguna manera, esta categoría de tensión central, y sus componentes hacen parte de la arquitectura de los marcos interpretativos desplegados, refiere a cada elemento del mapa de tensiones que se despliega, $y$, en esa medida, permite entender la forma, los ejes, las opciones y los alcances que adquiere la concreción del sistema de protección social que se observa en los programas estudiados. De este modo, los marcos interpretativos están en distancias y posiciones distintas respecto de los polos de tensión de la categoría principal.

Es así que unos están muy próximos y, de alguna manera, prácticamente adhieren y se superponen y(o) están asimilados (son lo mismo) con un polo de esta categoría central. Por ejemplo el polo Neoliberalismo corresponde con el PFC (o PFCM), y el polo Bienestarismo corresponde con el PFS (o PFM). En cambio, otro de los frames (el PFH o PFHM) se aproxima o más bien se orienta en el sentido de una de las posiciones de esta tensión, pero integra, mantiene elementos de la otra, de manera que está en un espacio no estrictamente equidistante, ya que se observa notoriamente que gravita en relación a uno de ellos (Bienestarismo), 
pero, a la vez, no se posiciona en la posición contraria al otro de los polos.

Podemos, de esta manera, observar que el PFH, y, con ello, la referencia principal que está en la concreción del programa, se ubica en una posición de cierta distancia de la tensión principal y con una determinada inclinación y, a la vez, con una determinada integración de elementos de la posición contraria de la que se inclina (Neoliberalismo). Así, cada una de las referencias centrales del PFH mantiene un vínculo, aunque no próximo, y en ningún caso hay asimilación con uno de estos polos, a la vez que integra, mantiene, no se tensiona (negocia o quizás ha debido negociar) con el polo contrario del que se inclina.

\section{DISCUSIÓN}

Un primer elemento a destacar es que, si bien existen consensos amplios respecto de los objetivos de la protección social, es que las políticas públicas resultan de la compleja trama que involucra, entre otros, las ideologías dominantes, los valores socialmente consensuados, los conflictos de intereses, los arreglos institucionales, los conocimientos y técnicas de actuación propios a cada sector. El diseño de políticas públicas está, en consecuencia, referido a estos factores, pero también va a ir cambiando según los contextos sociales, económicos, culturales y políticos. Son lo que denominamos, a grandes rasgos, las orientaciones de las políticas públicas. Sin embargo, las políticas públicas específicas pueden orientarse por más de un modelo explicativo, conformando sistemas alternativos que responden a sus exigencias particulares, donde el análisis de las políticas no sólo está cruzado por dichas orientaciones, sino que también por aspectos técnicos específicos que dan fuerza y sustento a esas orientaciones y se transforman en argumentos para el debate público sobre las opciones de cambio de políticas.

Los conflictos de frames a nivel meta (in- ter-casos), que se desprenden tanto de la Reforma Previsional como de Chile Solidario, y que son comunes a ambos, muestran las diferencias en las orientaciones de las políticas sobre protección social. Este conflicto genera estrategias de negociación que, finalmente, sitúan la respuesta hegemónica sobre la protección social más cercana al polo bienestarista, pero no completamente anclada en este. Efectivamente, lo que define la concreción del Marco Interpretativo Principal no es sólo la orientación valórica y de creencias, sino que existen aspectos técnicos que refuerzan, en el debate, la necesidad de realizar las reformas. Las opciones de cambio de políticas, sustentadas en el Marco Interpretativo Principal y Complementario están basadas, alternativamente en argumentos que provienen de un diagnóstico que es constituido en un problema público cuya solución difiere según el marco interpretativo adoptado. Respecto del Marco Interpretativo Principal intra casos, en el caso de la Reforma Previsional, por ejemplo, se resalta la necesidad de generar un piso de protección social garantizado, en un sistema mixto que articule tanto prestaciones contributivas como no contributivas, en tanto Chile Solidario no refiere a mecanismos de solidaridad y es no contributivo en esencia. La referencia a las inequidades en el ciclo vital son claras en la Reforma Previsional, no así en Chile Solidario, donde la noción de Sistema de Protección Social es aún incipiente.

Un segundo aspecto a destacar es que las políticas públicas, en tanto herramientas y acción, tienen una dimensión material y otra simbólica, como herramientas de cambio que se articulan con las formas de pensar de una sociedad e impactan en las condiciones de vida de las personas. En este sentido, las políticas públicas construyen y reconstruyen las legitimidades sociales sobre los cambios necesarios o el mantenimiento del status quo, sobre lo que es justo o injusto, los grupos que deben ser favorecidos, buscando acciones que permitan construir o mantener la cohesión social, a 
través de la articulación de un discurso coherente para el conjunto de la sociedad, que dote a las acciones públicas de legitimidad ante la ciudadanía.

De este modo, en el análisis intra e inter-casos, se observa que el Marco Interpretativo Principal refiere a la necesidad de realizar cambios en virtud de la equidad, para favorecer a los grupos más pobres, apelando a la necesidad de generar cambios no sólo en la provisión de bienes y servicios para estos sectores, sino también en la necesidad de cambios institucionales que generen un Sistema de Protección Social basado en derechos, que refuerce la cohesión social, en un contexto marcado por la desigualdad. En tanto, el Marco Interpretativo Complementario no se vincula con la idea de Sistema ni con la de derechos, sino solamente con la necesidad de favorecer a los grupos más pobres.

El Marco Interpretativo Complementario Meta, tanto en el análisis intra e inter- casos, es reflejo de la lógica de las políticas compensatorias como políticas privilegiadas de los gobiernos, especialmente en los 80. Esta visión no impulsa una reestructuración de la política social en forma integrada, implicada en un Sistema de Protección Social que contiene la necesidad de una reforma integral del Estado en ^ este campo, dirigida básicamente a recuperar : su legitimidad mediante políticas redistributi育 vas de carácter estructural.

Un tercer aspecto refiere a que, en el anáisis axial y selectivo de los casos, no existen conflictos de frames respecto de la necesidad de cambio en las políticas sociales. La necesidad de protección ante los riesgos y vulnerabilidades, a lo largo del ciclo vital, que sustenta el Sistema de Protección Social chileno, genera consenso. No así las soluciones, por lo que, por ejemplo, en el caso del análisis intra-casos de Chile Solidario, la respuesta del Marco Interpretativo Principal deja muchos temas sin consensos amplios, reflejados en las diferencias de diagnóstico y soluciones de políticas del Marco Interpretativos Complementario y en el caso de la Reforma Previsional del Marco Interpretativo Secundario. Ello probablemente hará surgir cambios en ambas reformas en escenario de reconstitución de fuerzas políticas.

Un cuarto elemento es que el Marco Interpretativo Secundario aparece cuando ya existe una trayectoria de reformas sociales y un contexto de impulso al Sistema de Protección Social basado en Derechos. Existe una evolución, un cambio en la discusión respecto del derecho a la protección social que puede atribuirse a la trayectoria de la protección social entre 2000 y 2008. De este modo, en Chile Solidario, hay muy poca discusión al respecto. No así en la Reforma Previsional, donde hay una comunidad interpretativa en el polo bienestarista y de universalización de derechos, que apela a cambios estructurales del sistema previsional. Esta es una discusión incipiente, que actualmente revive con más fuerza al haber cuestionamientos al modelo dual de provisión de servicios y a la necesidad de reformas o de modelo, que se encaminen a enfrentar las desigualdades sociales. En ese contexto, la dimensión de la ciudadanía y el bienestar, son asuntos que han sido enfrentados de manera paulatina e incremental en agenda social. Las solucione propuestas, tanto en Chile Solidario como en Reforma Previsional, desprendidas de los Marcos Interpretativos Principales Meta, mantienen importantes ribetes de continuidad con el diseño de la protección social en el primer ciclo de gobiernos de la coalición concertacionista (1990-2000).

En quinto lugar, de los antecedentes presentados se desprende que el conflicto principal refiere a la instalación de derechos en el Sistema de Protección Social, que refleja, por una parte, la tensión entre visiones más bien centradas en la protección, donde los derechos no son una variable central sino el aseguramiento de ingresos, y, por otra parte, la visión más centrada en el bienestar, con políticas universales, fundadas en derechos. Las discusiones sobre ¿Cuál es el piso mínimo de aseguramiento de derechos? ¿Pondremos un mínimo 
o un máximo y qué impactos financieros se derivan? ¿Cómo medir y disminuir las brechas sociales en términos de garantizar el acceso a prestaciones sociales? ¿Qué acuerdos o pactos políticos se requieren para definir los derechos que se garantizarán a la población? ¿Y a quienes se les garantizarán esos derechos? Estas y otras son preguntas aún abiertas, y las tensiones sobre las vías para responderlas van a reflejarse en el debate sobre qué tipo de políticas sociales generar en Chile y qué rol van a cumplir los derechos, no ya como mecanismos discursivos, sino con condiciones de exigibilidad.

Finalmente, este estudio constituye una primera aproximación al rol de las ideas, valores y creencias en la constitución del Sistema de Protección Social chileno, en una línea de análisis de políticas públicas que requiere una mayor densidad empírica para su consolidación en el país.

Recebido para publicação em 03 de fevereiro de 2016 Aceito em 20 de novembro de 2016

\section{REFERENCIAS}

ÁLVAREZ, Sonia (2005). Los discursos minimistas sobre las necesidades básicas y los umbrales de ciudadanía como reproductores de la pobreza. In: ÁLVAREZ, Sonia (Comp.). Trabajo y producción de la pobreza en Latinoamérica y el Caribe: estructuras, discursos y actores. Buenos Aires: CLACSO, p. 239-274.

ARENAS DE MESA, Alberto. Historia de la reforma previsional: una experiencia exitosa de política pública en democracia. Santiago: Oficina Internacional del Trabajo, 2010.139 p.

ARRIAGADA, Irma. Cambios de las políticas sociales: políticas de género y familia. Santiago de Chile: CEPAL.2006. (Serie Políticas Sociales, 119).

BARBA, Carlos. Los enfoques latinoamericanos de la política social: más allá del consenso de Washington. Revista Espiral, México, v. XI, n. 31, p. 85-130, 2004.

BARRIENTOS, Armando. Latin America: towards a liberal-informal welfare regime. In: GOUGH, I.; WOOD, G.(Eds.). Insecurity and Welfare Regimes in Asia, Africa and LatinAmerica: social policy in development contexts. Cambridge: Cambridge University Press, 2004. p.121-168.

CECCHINI, Simone; MARTÍNEZ, Rodrigo. Protección social inclusiva en América Latina. Una mirada integral, un enfoque de derechos. Santiago de Chile: CEPAL, 2011. $284 \mathrm{p}$.

COMISIÓN ECONÓMICA PARA AMÉRICA LATINA Y EL CARIBE (CEPAL). La hora de la igualdad. Brechas por cerrar, caminos por abrir. Santiago de Chile: CEPAL, 2010. 289 p.

CIMOLI, M. et al. Las Américas en la Encrucijada. Hacia la reinserción del trabajo digno en la agenda del desarrollo. New York: Carnegie Council on Ethics and International
Affairs, 2005.38 p. (Serie Foro globalización y desarrollo). Disponible em: < http:/www.policyinnovations.org/ideas/ policy_library/data/01172/_res/id=sa_File1/ > .Acceso en: 10 dez. 2015

CHARMAZ, Kathy. Constructing grounded theory. A practical guide through qualitative analysis. London: Sage Publications, 2006. 224p.

CORTÉS, Rosalía. The contemporary social policy debate in Latin America. Social Global Policy, [S.1], v. 8, n.1, p.109-114, 2008.

DEACON, Bob. From 'safety nets' back to 'universal social provision' Is the global tide turning?. Global Social Policy, [S.l], v. 5, n. 1, p. 19-28, 2005.

DELAMAZA, Gonzalo. Políticas públicas y sociedad civil en Chile: el caso de las políticas sociales (1990-2004). Política, [S.l], n. 3, 2004, p.105-148, 2004.

DÉLANO, Manuel. Reforma Previsional en Chile: protección social para todos. Santiago de Chile: Oficina Internacional del trabajo, $2010.152 \mathrm{p}$.

DE LEÓN, Peter. The historical roots of the field In: MORAN, M.; REIN, M.; GOODIN, R.E. (Eds.). The Oxford handbook of public policy. Oxford: Oxford University Press, 2006. p. 39-57.

DRAIBE Sonia; RIESCO, Manuel. Latin America: A new developmental Welfare State in the making? In: RIESCO, M. (Ed.). Latin American developmental Welfare State model in the making?. New York: Palgrave, MacMillan, 2007. p. 21-113.

ESTRADA ÁLVAREZ, Jairo. Sobre la metamorfosis del proyecto económico neoliberal. Revista de actualidad y análisis político, Colombia, 2004. Disponible em: < http:// www.cronicon.net/paginas/Documentos/paq2/No.27. pdf $>$. Acceso en: 17 feb. 2017

FILGUEIRA, Fernando. Cohesión, riesgo y arquitectura de protección social en América Latina. CEPAL, 2007. (Serie políticas sociales, 135).

FISCHER Frank. Reframing public policy. Discursive politics and deliberative practices, Oxford: Oxford University Press, 2003. 278p.

FORESTER., John. Learning from practice stories: the priority of practical judgment. In FISCHER,F.; FORESTER, J (Ed.). The argumentative turn in policy analysis and planning, Durham and London: Duke University Press, 1993. p.186-212.

GOMARIZ, Enrique. Sistema político y políticas públicas en América Latina. Revista Reforma y Democracia,[S.1], n. 38, p. 91-112, 2007.

HAJER, Marteen. Preface. In: HAJER, M.; WANEGAAR H. (Eds.). Deliberative policy analysis. Understanding governance in the network society. Cambridge: Cambridge University Press, 2003. p.xiii-xv.

HOWLETT, Michael; CASHORE, Benjamin. Re-Visiting the New Orthodoxy of policy dynamics: the dependent variable and re-aggregation problems in the study of policy change. Canadian Political Science Review, Canada, v. 1, n. 2, p. 50-62, 2007.

INTERNATIONAL LABOUR ORGANIZATION (ILO). Social protection floor for a fair and inclusive globalization. Report of the social protection floor advisory group. Geneva, International Labour Office, 2011. 117p

KLIKSBERG, Bernardo. Diez falacias sobre los problemas sociales en América Latina. Revista Reforma y Democracia [S.1], v.1, n. 19, p.1-22, 2001.

MARTIN, María Pía. Protección social, igualdad y construcción de un Estado de Bienestar en Chile Revista de Asuntos Públicos, [S.1], n. 718, junio 2009.

MARTNER, Gonzalo. Reformas a la protección y seguridad 
social en Chile. Políticas Públicas, Maranhão, v. 3, n. 1, p. $61-94,2010$

MARTÍNEZ FRANZONI, Juliana. Regímenes de bienestar en América Latina. Madrid: Fundación Carolina, 2007. $118 \mathrm{p}$

MOLYNEUX, Maxine. "Change and continuity in social protection in Latin America: mothers at the service of the state?" Geneva: United Nations Research Institute for Social Development (UNRISD), 2007.

MULLER, Pierre. L'Analyse cognitive des politiques publiques: vers une sociologie politique de l'action publique. Revue Française de Science Politique, [S.1], v. 50, n. 2 , p. $189-207,2000$.

PALMA, Julieta; URZÚA, Raúl. Políticas contra la pobreza y ciudadanía social: el caso de Chile Solidario. Santiago de Chile: Departamento de Políticas Públicas, 2005. (Colección Políticas Sociales, 12).

RACZYNSKI, Dagmar. Sistema Chile solidario y la protección social de Chile -. lecciones del presente y agenda para el futuro. São Paulo; Santiago de Chile: Instituto Fernando Henrique Cardoso, 2008. $52 \mathrm{f}$.

RAWLINGS, Laura. A new approach to social assistance: latin America's 157 experience with conditional cash transfer programs. World Bank, 2004. (Social Protection Discussion Paper Series, 0416).

RIBE, Helena; ROBALINO, David; WALKER, Ian. Achieving effective social protection for all in Latin American and the Caribbean. From right to reality. Washington: World Bank, 2010. $140 \mathrm{p}$

SABATIER, Paul A.; SCHLAGER, Edella. Les approches cognitives des politiques publiques: perspectives américaines. Revue Française de Science Politique, [S.l], v. 50, n. 2, p. 209-234, 2000 .

SCHMIDT, Vivien; RADAELLI, Claudio. Policy change and discourse in Europe: conceptual and methodological issues. West European Politics, Inglaterra, v. 27, n. 2, p. 183-210, 2004.

SCHÖN Donald; REIN Martin.Frame Reflection: toward a resolution of intractable policy controversies. New York: Basic Books, 1994. 247 p.
SERRANO, Claudia. La política social en la globalización. Programas de protección en América Latina. Santiago de Chile: CEPAL, 2005.

SOJO, Ana. Vulnerabilidad social, aseguramiento y diversificación de riesgos en América Latina y el Caribe. Revista de la CEPAL, Santiago de Chile, n. 80, ago. 2003

SOTELSEK, Daniel. Exclusión social y pobreza en América Latina. Revista Española del Tercer Sector, Espanha, n. 5, p. 111-146, ene./abr. 2007.

STOLOWICZ, Beatriz. La tercera vía en América Latina: de la crisis intelectual al fracaso político. In: ESTRADA, Jairo (Ed.). Intelectuales, tecnócratas y reformas neoliberales en América Latina. Bogotá: Universidad Nacional de Colombia; Facultad de Derecho, 2005. p. 55-81.

STERN, Phyllis Noerager. On solid ground: essential properties of growing grounded theory. In: BRYANT A.; CHARMAZ, K. (Eds.). Handbook of grounded theory. London: Sage Publications, 2007. p.114-126.

STRAUSS, Anselm; CORBIN, Juliet. Bases de la investigación cualitativa. Técnicas y procedimientos para desarrollar la teoría fundamentada. Colombia: Universidad de Antioquia, 2002. $341 \mathrm{p}$.

TRINIDAD, Antonio; CARRERO, Virginia; SORIANO, Rosa María. Teoría fundamentada (grounded theory). La construcción de la teoría a través del análisis interpretacional. Madrid: Centro de Investigaciones Sociológicas, $2006.174 \mathrm{p}$.

VILLATORO, Pablo. Las transferencias condicionadas en América Latina: luces y sombras. Santiago de Chile: CEPAL, 2007.

VERLOO, Mieke. Policy frames and implementation problems: the case of gender mainstreaming. Part B: proposal description of rtd projects. NewYork, 2002. Disponible em: <www.mageeq.net>. Acceso en: 10 jul. 2010 .

WILLIAMSON, John. No hay consenso. Finanzas y desarrollo, Washington, v. 40, n. 3, p. 10-13, 2003

YIN, Robert. The case study anthology. USA: Sage Publications, 2004. $271 \mathrm{p}$. 


\section{POLICY OF WELFARE IN NEOLIBERAL CONTEXTS: tensions of the Chilean model}

\author{
María Pía Martin \\ Jaime Alfaro
}

Contemporary Latin American societies have been pressured by conflicts between multiple views about the articulation between economic growth and social policies. This article presents the analysis of interpretive frameworks that, from the constructivist perspective, permit to deepen the symbolic and representative dimension of the policies. From the theoretical reflections and analyses about the visions of social protection in parliamentary discussions, in the case of Chile, the purpose of this study is to bring a perspective of analysis that permits to inquire about the processes social policies change. In addition, this study aims to demonstrate how the axis, in the ideas, offers a better comprehension of the stability and change of social policies.

KEYwORDS: Social Protection. Welfare state. Neoliberalism. Analysis of interpretive frameworks. Public Policy.

\section{PROTECTION SOCIALE DANS DES CONTEXTES NEOLIBERAUX: tensions du modèle chilien}

\author{
María Pía Martin \\ Jaime Alfaro
}

\begin{abstract}
Les sociétés contemporaines d'Amérique latine ont été mises sous tension par les conflits entre les multiples points de vue sur la relation entre la croissance économique et les politiques sociales. Cet article présente l'analyse des cadres interprétatifs que, depuis le approche constructiviste, permet de comprendre la dimension symbolique et interprétative des politiques. A partir des considérations théoriques et des analyses sur les points de vue sur la protection sociale dans le cas du Chili, le but est de fournir une perspective analytique qui permet d'étudier les processus de changement dans la politique sociale, et de montrer comment l'axe dans le idées permet une meilleure compréhension de la stabilité et le changement dans les politiques sociales.
\end{abstract}

Mots-CLÉs: Protection Sociale. l’État-providence. Le néolibéralisme. L’analyse des Cadres Interprétatifs. Les Politiques Publiques. 
